\title{
Non-equilibrium fluctuations in frictional granular motor: experiments and kinetic theory
}

\author{
Andrea Gnol粎 \\ Istituto dei Sistemi Complessi - CNR and Dipartimento di Fisica, \\ Università "Sapienza", p.le A. Moro 2, 00185 Rome, Italy and \\ Istituto dei Sistemi Complessi - CNR, via del Fosso del Cavaliere 100, 00133 Rome, Italy \\ Alessandro Sarracind \\ Istituto dei Sistemi Complessi - CNR and Dipartimento di Fisica, \\ Università "Sapienza", p.le A. Moro 2, 00185 Rome, Italy \\ Alberto Petris \\ Istituto dei Sistemi Complessi - CNR, via del Fosso del Cavaliere 100, 00133 Rome, Italy
}

\begin{abstract}
We report the study of a new experimental granular Brownian motor, inspired to the one published in [Phys. Rev. Lett. 104, 248001 (2010)], but different in some ingredients. As in that previous work, the motor is constituted by a rotating pawl whose surfaces break the rotation-inversion symmetry through alternated patches of different inelasticity, immersed in a gas of granular particles. The main novelty of our experimental setup is in the orientation of the main axis, which is parallel to the (vertical) direction of shaking of the granular fluid, guaranteeing an isotropic distribution for the velocities of colliding grains, characterized by a variance $v_{0}^{2}$. We also keep the granular system diluted, in order to compare with Boltzmann-equation-based kinetic theory. In agreement with theory, we observe for the first time the crucial role of Coulomb friction which induces two main regimes: (i) rare collisions $(\mathrm{RC})$, with an average drift $\langle\omega\rangle \sim v_{0}^{3}$, and (ii) frequent collisions (FC), with $\langle\omega\rangle \sim v_{0}$. We also study the fluctuations of the angle spanned in a large time interval, $\Delta \theta$, which in the FC regime is proportional to the work done upon the motor. We observe that the Fluctuation Relation is satisfied with a slope which weakly depends on the relative collision frequency.

PACS numbers: 45.70.-n,05.40.-a
\end{abstract}

\section{INTRODUCTION}

Brownian motors (BM) are devices that can rectify thermal fluctuations, in order to perform work against external loads [1, 2]. The basic underlying mechanism relies on the presence of non-equilibrium conditions, breaking the time-reversal symmetry in the dynamics, together with some spatial anisotropy, which allows unidirectional motion. Although these general constraints are clearly understood [3], many open questions remain to be answered, concerning the several different mechanisms for the realization of such devices. For instance, given a particular shape of the probe, the prediction of the drift direction is far from obvious, in particular if several sources of dissipation are present in the system, inducing competitive effects.

Recent years have seen an increasing wide interest on Brownian motors directly inspired to the original setup of the Feynman's ratchet [3]. In these "collisional Brownian motors" (CBM) fluctuations are induced by the collisions of an asymmetric probe with particles of molecular flu-

\footnotetext{
* andrea.gnoli@isc.cnr.it

$\dagger$ alessandro.sarracino@roma1.infn.it

$\ddagger$ andrea.puglisi@roma1.infn.it

$\S$ alberto.petri@isc.cnr.it
}

ids at different temperatures [4 $[6]$. Since the presence of dissipation is a fundamental ingredient to induce nonequilibrium conditions, a natural framework where these kinds of systems have been studied is the realm of granular media, where interactions do not conserve energy due to inelasticity. Several experimental [7-10] and theoretical results [11 14] have been obtained for these systems.

More recently, another source of dissipation has been shown to play an important role in the dynamics of CBM: the Coulomb (or dry) friction [15 17]. Its main effect is the introduction of two dynamical regimes, where the behavior of the systems is dominated by collisions or friction, respectively. More surprisingly, it has also been shown that the Coulomb friction itself can be sufficient to drive a motor effect, even if the probe is in contact with a single molecular fluid at equilibrium [17, 18]. The role of friction has also been studied in other systems showing motor effects [19, 20], where fluctuations are introduced by noise terms, which are not related to particle collisions.

The context of granular systems also paves the way to the realization of experiments aimed at validating some important general relations derived for nonequilibrium systems, such as the Fluctuation Relation [21] or the Hatano-Sasa relation [22]. In particular, in granular systems, where noise and time-scale separation are often not fully under control and where some coarse-grained on the 
accessible quantities is present, the experimental study of these relations is very useful to assess such results in more general situations 23 25].

In this paper we consider a new experimental setup for a frictional granular CBM, in order to get closer to conditions where kinetic theory can be applied. Moreover, at variance with previous studies, we also take into account the presence of Coulomb friction which induces interesting behaviors. This allows us to compare experimental results with analytical predictions of kinetic theory. Furthermore, we focus on the study of the non-equilibrium fluctuations of the spanned angle in a time interval, which is related to the work done by the CBM. Our findings suggest that a symmetry relation for these fluctuations is verified in our system, in agreement with previous results for similar experiments [23].

\section{SETUP}

The two main components of our setup are the granular gas and the rotor, see Fig. 11 for visual explanation. The granular gas is made of $N=50$ spheres of polyoxymethylene (diameter $d=6 \mathrm{~mm}$ and mass $m=0.15 \mathrm{~g}$ ) contained in a polymethyl-methacrylate (PMMA) cylinder of circular base, with area $A \approx 6.36 \times 10^{3} \mathrm{~mm}^{2}$ and maximum height $70 \mathrm{~mm}$. The cylinder is shaken with a sinusoidal signal at $53 \mathrm{~Hz}$ and variable amplitude, which is measured by the maximum acceleration $\Gamma=a_{\max } / G$ rescaled by the gravity acceleration $G$. The velocity distribution of the spheres on the plane perpendicular to the rotation axis is obtained by particle tracking via a fast camera (see [26] for details on the procedure) and is fairly approximated by a Gaussian,

$$
p_{g}(v) \sim e^{-v_{x}^{2} /\left(2 v_{0}^{2}\right)},
$$

where the "thermal" velocity $v_{0}$ has been introduced and $v_{x}$ may be replaced by $v_{y}$ because the system is isotropic on the $\hat{x y}$ plane. Small deviations from the Gaussian are observed but are neglected for the purpose of this study; see [17] for details. We have changed $\Gamma$ from 5 to 21 , finding for $v_{0}$ values from $120 \mathrm{~mm}^{2} \mathrm{~s}^{-2}$ to 500 $\mathrm{mm}^{2} \mathrm{~s}^{-2}$. The vertical average profile of the gas density is close to the so-called "leading order" distribution [27]: $n_{0}(z)=\frac{N G}{A v_{0}^{2}} \exp \left(\frac{-G}{v_{0}^{2}} z\right)$, where $z$ is the coordinate of the vertical axis. This is sufficient to evaluate the average density surrounding the rotator, estimated to be $n=$ $n_{0}\left(z^{*}\right)$ where $z^{*}$ is the mid-heigth of the rotator.

Suspended into the gas, we have a pawl (also called "rotator") rotating around a vertical axis. The rotator is a PMMA cylinder with a rectangular base (see lowerright inset of Fig. 1) of perimeter $S$, height $h$ and total surface of the sides $\Sigma=S h=1.2 \times 10^{-3} \mathrm{~mm}^{2}$. The rotator (including its axis) has mass $M=6.49 \mathrm{~g}$ and moment of inertia $I=353 \mathrm{~g} \mathrm{~mm}^{2}$. The axis of the rotator is suspended to two spheres bearing. The position vs time of the rotator is recorded by an angular encoder (Avago
Technologies, model AEDA-330), enclosing the bearings. In Fig. 11 top-right inset, the definition of some quantities useful for the theory can be found. The study of the Brownian motor phenomenon is obtained by applying insulating tape to the rotator, partially covering its two largest surfaces, see lower-right inset of Fig. 1 for an explanation. Turning upside-down the rotator allows one to invert its chirality, defining two possible orientations which we call "right" and "left".

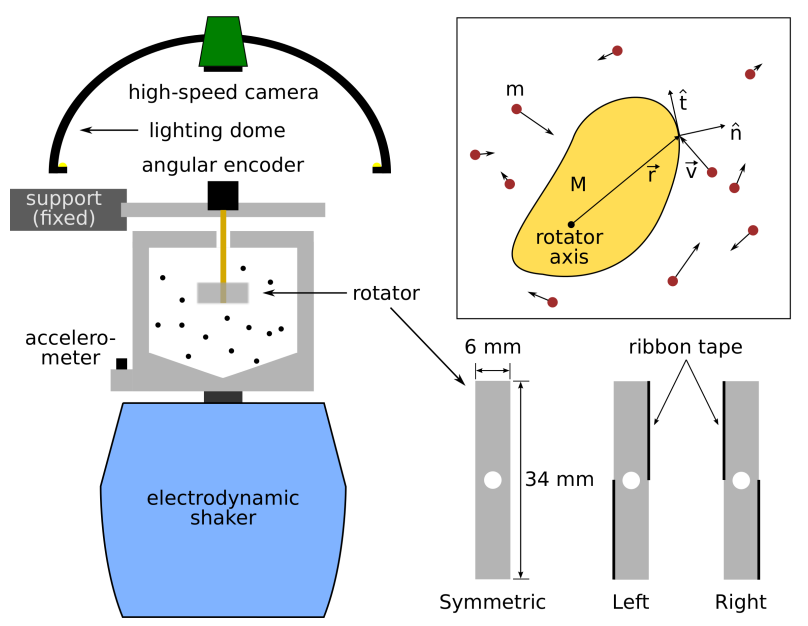

FIG. 1. Color online: the experimental setup

A close analysis of the dynamics of the rotator shows that the angular velocity $\omega$ is well described by the following equation of motion:

$$
\dot{\omega}(t)=-\Delta \sigma[\omega(t)]-\gamma_{a} \omega(t)+\eta_{\text {coll }}(t)
$$

where $\Delta=F_{\text {frict }} / I=38 \pm 4 \mathrm{~s}^{-2}$ is the frictional force rescaled by inertia, $\gamma_{a}=6 \pm 1 \mathrm{~s}^{-1}$ is some viscous damping rate related perhaps to air or to other dissipations in the bearings, and $\eta_{\text {coll }}(t)$ is the random force due to collisions with the granular gas particles. The granular gas itself is stationary and (roughly) homogeneous with a mean free path proportional to $\lambda=(n \Sigma)^{-1}$. The pawl is further characterized by its symmetric shape factor $\left\langle g^{2}\right\rangle_{\text {surf }}=1.51$ (see [13] for details), with \langle\rangle$_{\text {surf }}$ being a uniform average over the surface of the object parallel to the rotation axis and $g(s)=\frac{\mathbf{r}(s) \cdot \hat{t}(s)}{R_{I}}$ with $\hat{t}(s)=\hat{z} \times \hat{n}(s)$ which is the unit vector tangent to the surface at the point $\mathbf{r}(s)$, and $\hat{n}(s)$ is the unit vector perpendicular to the surface at that point. We have also introduced the radius of inertia $R_{I}=\sqrt{I / M}$ of the rotator. We refer to the top-right inset of Fig. 1 for a visual explanation of symbols. The restitution coefficient between spheres and the pawl has been measured to be $\alpha_{+} \approx 0.67$ on the PMMA naked surface and $\alpha_{-} \approx 0.35$ on the tape-covered face.

It is useful to introduce the "equipartition" angular velocity $\omega_{0}=v_{0} \epsilon / R_{I}$ where $\epsilon=\sqrt{\frac{m}{M}}$. Note that, because of inelastic collisions and frictional dissipations, the rotator does not satisfy equipartition and $\omega_{0}$ is only a useful 
reference value. It is natural to adimensionalize the rotator angular velocity defining $\Omega=\frac{\omega}{\omega_{0}}$.

\section{THEORY}

The single particle probability density function (pdf) $p(\omega, t)$ for the angular velocity of the rotator is fully described, under the assumption of diluteness which guarantees Molecular Chaos, by the following linear Boltzmann equation [13, 15, 17]

$$
\begin{aligned}
\partial_{t} p(\omega, t) & =\partial_{\omega}\left[\left(\Delta \sigma(\omega)+\gamma_{a} \omega\right) p(\omega, t)\right]+J\left[p, p_{g}\right] \\
J\left[p, p_{g}\right] & =\int d \omega^{\prime} W\left(\omega \mid \omega^{\prime}\right) p\left(\omega^{\prime}, t\right)-p(\omega, t) f_{c}(\omega), \\
f_{c}(\omega) & =\int d \omega^{\prime} W\left(\omega^{\prime} \mid \omega\right) \\
W\left(\omega^{\prime} \mid \omega\right) & =\rho S \int \frac{d s}{S} \int d \mathbf{v} p_{g}(\mathbf{v}) \Theta[(\mathbf{V}(s)-\mathbf{v}) \cdot \hat{n}] \times \\
|(\mathbf{V}(s)-\mathbf{v}) \cdot \hat{n}| \delta\left[\omega^{\prime}-\omega-\Delta \omega(s)\right], & \\
\Delta \omega(s) & =(1+\alpha) \frac{[\mathbf{V}(s)-\mathbf{v}] \cdot \hat{n}}{R_{I}} \frac{g(s) \epsilon^{2}}{1+\epsilon^{2} g(s)^{2}},
\end{aligned}
$$

where we introduce the rates $W\left(\omega^{\prime} \mid \omega\right)$ for the transition $\omega \rightarrow \omega^{\prime}$, the velocity-dependent collision frequency $f_{c}(\omega)$, the pdf for the gas particle velocities $p_{g}(\mathbf{v})$ and the socalled kinematic constraint in the form of Heaviside step function $\Theta[(\mathbf{V}-\mathbf{v}) \cdot \hat{n}]$ which enforces the kinematic condition necessary for impact. Here $\mathbf{V}(s)=\omega \hat{z} \times \mathbf{r}(s)$ is the linear velocity of the rotator at the point of impact $\mathbf{r}(s)$. The collision rule is implemented by Eq. (3e [13].

An estimate of the ratio between the stopping time due to dissipation (dominated by dry friction) $\tau_{\Delta} \sim \frac{\omega_{0}}{\Delta}$ and the collisional time $\tau_{c} \sim \frac{1}{n \Sigma v_{0}}$ is given by the parameter

$$
\beta^{-1}=\frac{\epsilon n \Sigma v_{0}^{2}}{\sqrt{2} \pi R_{I} \Delta} \approx \frac{\tau_{\Delta}}{\tau_{c}} .
$$

This parameter controls the transition from a regime (at $\left.\beta^{-1} \ll 1\right)$ with fast stopping due to dissipation, called $\mathrm{RC}$ (rare collisions), and a regime (at $\beta^{-1} \gg 1$ ) with the rotator always in motion, continuously perturbed by collisions, called FC (frequent collisions).

When the mass of the rotator is large with respect to the mass of the granular gas particles, collisions are small perturbations to $\omega(t)$ (see Eq. (3e)). Then, it makes sense to expand Eq. (3) in powers of $\epsilon$ [12, 13]: by retaining only up to the second derivative, a Fokker-Planck equation is obtained. The basic result of this procedure is that the collisional noise in Eq. (2) is cast into the sum of a white noise $\eta(t)$ plus a viscous drag and a systematic force inducing the motor effect:

$$
\eta_{\text {coll }}(t) \rightarrow \eta(t)-\gamma_{g} \omega(t)+\tau_{\text {motor }},
$$

with $\langle\eta\rangle=0$ and $\left\langle\eta(t) \eta\left(t^{\prime}\right)\right\rangle=\Gamma_{g} \delta\left(t-t^{\prime}\right)$. The expression for $\gamma_{g}, \tau_{\text {motor }}$ and the amplitude of the noise $\Gamma_{g}$ have been obtained, for a generic asymmetric rotator in a dilute granular gas, in [13]. For our particular shape they read 15$]$

$$
\begin{aligned}
\gamma_{g} & =\sqrt{\frac{2}{\pi}} \lambda^{-1} \epsilon^{2} v_{0}\left\langle(1+\alpha) g^{2}\right\rangle_{\text {surf }}, \\
\tau_{\text {motor }} & =\gamma_{g} \sqrt{\frac{3 \pi}{32}} \frac{1}{\epsilon} \frac{\alpha_{+}-\alpha_{-}}{2+\alpha_{+}+\alpha_{-}}, \\
\Gamma_{g} & =(1+\alpha) \gamma_{g} \frac{\epsilon^{2}}{R_{I}^{2}} v_{0}^{2},
\end{aligned}
$$

with $\alpha=\left(\alpha_{+}+\alpha_{-}\right) / 2$. We want to highlight, here, a fundamental point concerning the collisional noise $\eta_{\text {coll }}$ : as Eq. (5) explicitly shows, such a noise is in general not white, and, even more importantly, it is not independent from the instantaneous velocity $\omega$. This makes sense, as it is the superposition of the variations of angular velocity due to collisions, which, as shown by Eq. (3e), depends on $\omega$. For this reason our model, described alternatively by Eq. (2) or Eq. (3), is very different from a model apparently similar - recently introduced in [20], as well as from other previous models [28].

In the FC limit, $\beta^{-1} \gg 1$, the Coulomb friction term and the external viscosity may be neglected, i.e.

$$
\gamma_{a} \omega+\Delta \sigma(\omega) \ll \gamma_{g} \omega
$$

so that Eq. (2) is cast into the much simpler form

$$
\dot{\omega}(t)=-\gamma_{g} \omega(t)+\eta(t)+\tau_{\text {motor }} .
$$

From such an equation one may estimate the average velocity of the Brownian motor to be [15, 16]

$$
\langle\omega\rangle=\frac{\tau_{\text {motor }}}{\gamma_{g}} .
$$

Another interesting observation follows from Eq. (10). It concerns the fluctuations $f(\Delta \theta)$ of the spanned angle in a time interval of length $\Delta t, \Delta \theta=\theta(t+\Delta t)-\theta(t)$ for any $t$ in the steady state. For the particularly simple linear Langevin case, Eq. (10), it can be shown that such fluctuations obey, for large $\Delta t$, the following Fluctuation Relation (FR) [29]:

$$
\phi(\Delta \theta)=\log \left[\frac{f(\Delta \theta)}{f(-\Delta \theta)}\right] \approx s \Delta \theta,
$$

with

$$
s=\frac{\gamma_{g} \tau_{m o t o r}}{\Gamma_{g}} \approx \frac{\tau_{m o t o r}}{\left\langle\omega^{2}\right\rangle}
$$

We mention that such an FR is closely related to the $\mathrm{FR}$ for the entropy produced in the time $\Delta t$, which in this system is approximated by the work done by the "Brownian motor force" $W \approx \tau_{\text {motor }} \Delta \theta$ divided by the "temperature" $\left\langle\omega^{2}\right\rangle[30]$.

In the $\mathrm{RC}$ regime, on the other side, one may assume that the probe's dynamics is a sequence of independent 
kicks received at zero velocity, resulting in the following formula for the adimensional average angular velocity [15]:

$$
\begin{aligned}
\langle\Omega\rangle & =q \beta^{-1} \\
q & =\frac{\sqrt{\pi}}{4}\left[\left(1+\alpha_{+}\right)^{2}-\left(1+\alpha_{-}\right)^{2}\right] \\
& \times\left(\frac{\tan ^{-1} \sqrt{\xi}}{\sqrt{\xi}}-\frac{1}{1+\xi}\right),
\end{aligned}
$$

where $\xi=m L^{2} /(4 I)$ and $L$ is the lenght of the pawl. We note that for the dimensional angular velocity this means $|\omega| \sim v_{0}^{3} \epsilon$. In the RC regime the behavior of $\phi(\Delta \theta)$ is unknown in principle. A FR for the entropy production certainly exists, but we are not aware of a simple relation between $\Delta \theta$ and the entropy produced in a given time interval.

\section{MOTOR EFFECT}

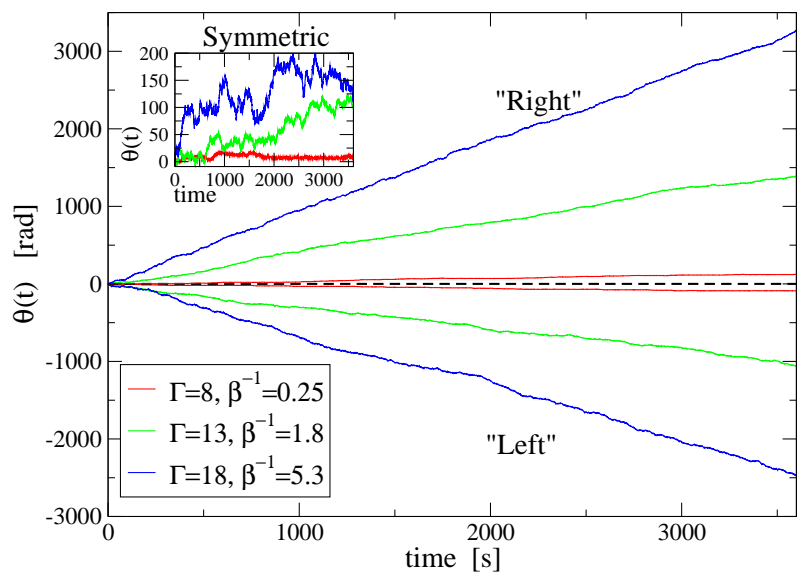

FIG. 2. Color online: trajectories with different shaking parameters and different chiralities. The inset shows the behavior of the symmetric (i.e., without tape) pawl.

In Figure 2 the evolution in time of the angle $\theta(t)$ spanned by the rotator is shown for different choices of the maximum shaking acceleration $\Gamma$ and different orientation of the asymmetry ("left", L, or "right", R). A measure of the "thermal" velocity of the particles, $v_{0}$, through the fast camera, allows one to determine $\beta^{-1}$ which estimates the relative relevance of collisions with respect to Coulomb friction. A steady drift, signaling the presence of Brownian motor effect, is observed both in the friction dominated regime $\left(\beta^{-1}<1\right)$ and in the collisions dominated regime $\left(\beta^{-1}>1\right)$. Turning the rotator upside down, i.e. changing its asymmetry from $L$ to $R$ or viceversa, inverts the sign of the drift: such an observation confirms that the observed drift is caused by the asymmetry of $\alpha$, as expected from the given theoretical arguments. A further confirmation that the observed drift is due to the surface heterogeneity comes from the study of the "symmetric" rotator, which has no patches of insulating tape: on the same timescale only a very weak drift is observed, much weaker than the one observed with the L- and R-type rotators. We impute such a weak bias measured with the symmetric rotator to imperfections of the experimental setup: any kind of asymmetry (e.g. not sufficiently precise vertical alignments, not perfectly circular profile of the container, etc.) may induce secondary motor effects.

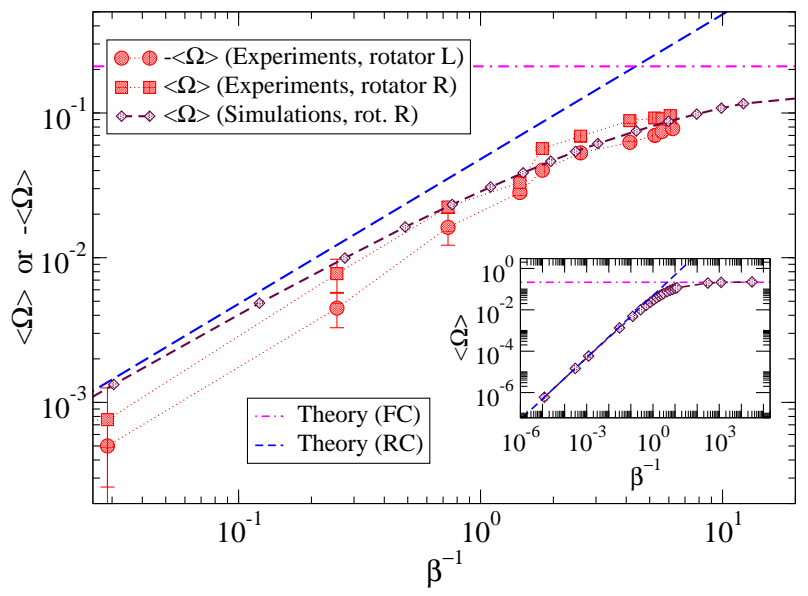

FIG. 3. Color online: Average angular velocity, rescaled by $\omega_{0}$, of the rotator: experiments, theory and simulations, for several values of $\beta^{-1}$ and both chiralities. In the inset only simulations are show, in order to appreciate the comparison with both RC and FC theoretical limits at very small and very large $\beta^{-1}$.

In Figure 3, the rescaled average angular velocity of the rotator, $\langle\Omega\rangle$, measured in experiments with both Land $\mathrm{R}$-type rotators, is shown as a function of $\beta^{-1}$. The velocity of the L-rotator is changed of sign for the purpose of a better visualization. In the same plot we have also shown the results of numerical simulations of Eq. (3) with the same parameters as in the experiment (only for the R-type rotator). Details about the simulation can be found in the Supplemental Material of ref. [17]. As a first observation, we highlight the quite good agreement between experimental and numerical data.

The thick blue dashed and magenta dot-dashed lines represent the theoretical predictions for the $\mathrm{RC}$ and $\mathrm{FC}$ limits, Eqs. (14) and (11) respectively. In the inset of the Figure we have displayed the results of the simulations on a much wider range of $\beta^{-1}$, in order to appreciate the agreement with the theoretical limits. The simulations teach us that such theoretical predictions for the RC limit (FC limit) are useful for quite small (large) values of $\beta^{-1}$. The experimentally accessible values of $\beta^{-1}$ appear to be at the crossover between the two regimes: nevertheless they span a sufficiently wide range, so that both the $\langle\Omega\rangle \sim \beta^{-1}$ behavior ( $\mathrm{RC}$ regime) and the trend toward saturation $\langle\omega\rangle \rightarrow$ const. (FC regime) can be iden- 
tified. We consider this to be the best comparison, up to our present knowledge, between experimental granular Brownian motors and kinetic theory. We mention that it is quite difficult to expand the accessible $\beta^{-1}$ range. Indeed exploring smaller values of $\beta^{-1}$ requires a considerable increase of the dry friction coefficient $\Delta$, which is not under our direct control; moreover, a large $\Delta$ may amplify non-ideal effects where the behavior of the spheres bearings do not follow the Coulomb law: such effects are already observed here (at small velocities small deviations are observed) and are likely responsible for the not perfect match with simulation results at small $\beta^{-1}$; we also notice that static friction is not considered here, but it could become relevant at large $\Delta$ [31]. The opposite limit, i.e. large values of $\beta^{-1}$, are even more difficult to be attained, since they would require a larger collision frequency: however the maximum acceleration of the shaker is a hard limit, while increasing the number of grains does not trivially produces the desired result, for two reasons: 1) higher densities correspond to a reduction of the average kinetic energy and 2) Molecular Chaos is only guaranteed at low density. Notwithstanding those limits, we believe that the results of Fig. 3 are already a quite good success of kinetic theory and make us claim that Eq. (3) is a fair description of the experimental setup.

\section{NON-EQUILIBRIUM FLUCTUATIONS}

(a)

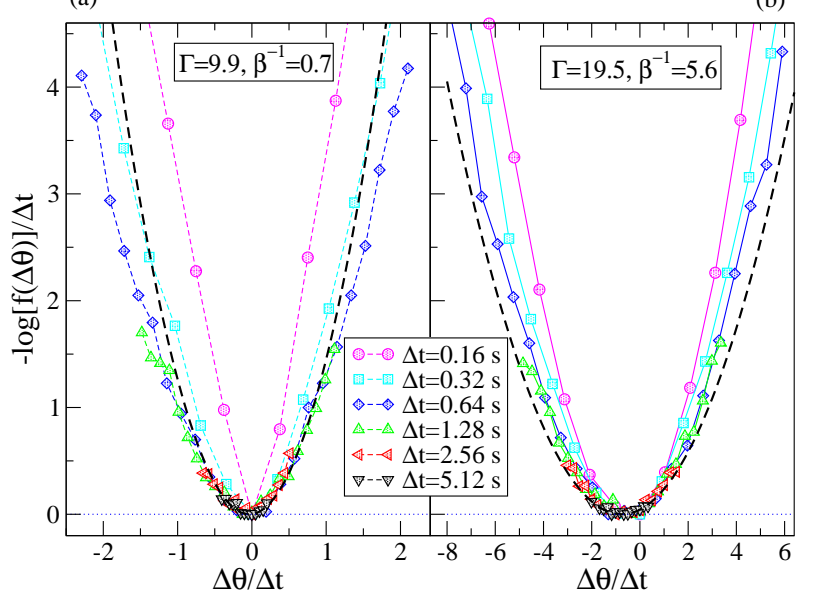

FIG. 4. Color online: Empirical large deviation function for the fluctuations of $\Delta \theta$ for two experiments in the $\mathrm{RC}$ regime (left) and in the FC regime (right), at several values of $\Delta t$. A small correction is operated to each pdf $f(\Delta \theta)$, normalizing it by its maximum value, before taking the logarithm, for the purpose of a better vertical alignment.

In Fig. 4 we have displayed the empirical large deviation rate $(\operatorname{ldr})-\log [f(\Delta \theta)] / \Delta t$ of the pdf $f(\Delta \theta)$ for different choices of the time window $\Delta t$, in two experiments with a small and a large value of $\beta^{-1}$. In both cases we have also superimposed a parabolic fit of the data at the largest available time. We mention that the characteristic time $\tau_{c}$ for the decay of the angular velocity autocorrelation, not shown here, is in the range $0.03-0.06 \mathrm{~s}$. As frequently observed [32, 33], the empirical large deviation rate tends to become independent from time only at very large $\Delta t \gg \tau_{c}$. Here we evaluate $\Delta t \sim 1 \mathrm{~s}$ as a minimum value before considering reached the large deviation limit. In both experiments we may appreciate deviations from the parabolic fit, i.e. slightly non-Gaussian tails, signaling that we are indeed probing the large deviations of the pdf of $\Delta \theta$.
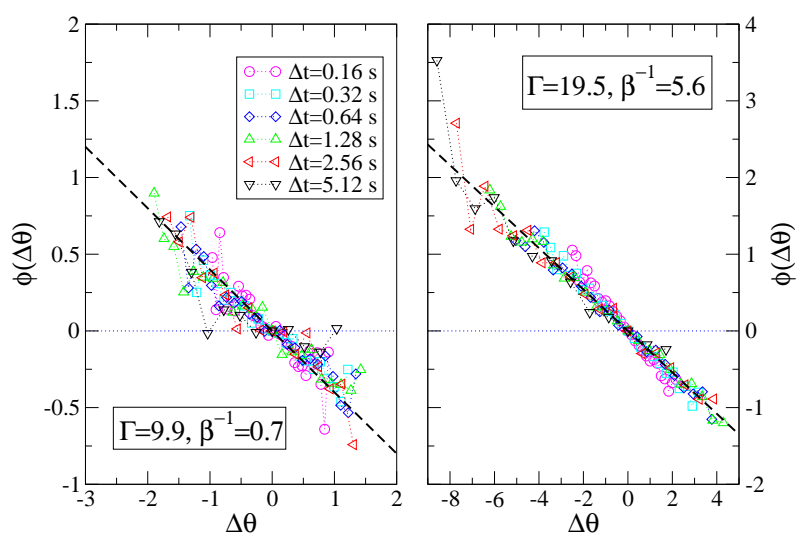

FIG. 5. Color online: Asymmetry function $\phi(\Delta \theta)$ for the pdfs of $\Delta \theta$ for two experiments in the $\mathrm{RC}$ regime (left) and in the $\mathrm{FC}$ regime (right), at several values of $\Delta t$.

The asymmetry function $\phi(\Delta \theta)=\log \left[\frac{f(\Delta \theta)}{f(-\Delta \theta)}\right]$ for the pdf of $\Delta \theta$ is shown, for the same two experiments, in Fig. 5. At values of $\Delta t$ large enough, but smaller than those necessary to achieve a stable large deviation rate, the asymmetry functions already display a linear behavior $\sim s \Delta \theta$ with a slope $s$ not dramatically changing with time. The values of the slope have been measured for all experiments and many values of $\Delta t$ and are reported in Fig. 6.

While confirming the direct observation done in Fig. [5, Fig. [6 clearly teaches us that the slope of the $\phi(\Delta \theta)$ becomes stable at times of the order $\Delta t \sim 0.3 \mathrm{~s}$. In the inset of Fig. 6 we have displayed the slope $s$ (from a constant fit of the main plot including values $\Delta t \geq 0.32 \mathrm{~s}$ ) vs. $\beta^{-1}$. The squared symbols joined by the green dashed line represent the result of formula (13). Many important comments are in order here: 1) for large values of $\beta^{-1}$, as expected, dry friction becomes negligible and - at the leading order - the system is described by Eq. (10), which is confirmed by the good agreement of the slope with Eq. (13); 2) at moderate and small values of $\beta^{-1}$ the "simplified" Langevin description of Eq. (10) is not expected to hold, and indeed discrepancy is found between experimental slopes of $\phi(\Delta \theta)$ and those predicted by Eq. (13); nevertheless 3) such experimental values of the slope appear to depend only weakly upon $\beta^{-1}$, so that 


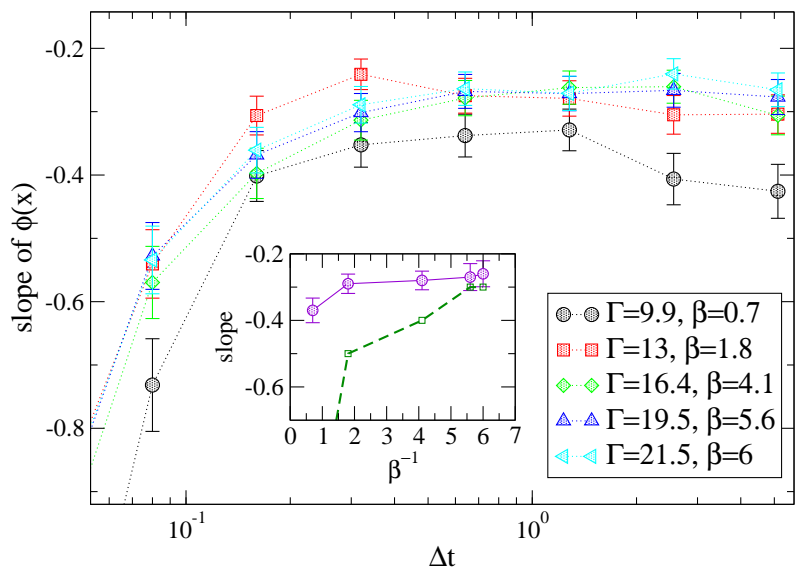

FIG. 6. Color online: Slopes of the asymmetry functions for several different experiments, as a function of $\Delta t$. The inset shows the value of the slope (the values of each experiments are averaged over the plateau visible in the main plot at large $\Delta t)$ as a function of $\beta^{-1}$. The squares joined by the green dashed line represent the result of formula (13).

they do not differ too much from the values at large $\beta^{-1}$. The last observation is an empirical fact which has not a simple explanation: at small values of $\beta^{-1}$ the noise felt by the rotator is discontinuous and the average drift can hardly be described as the effect of a continuous torque (as it is $\tau_{\text {motor }}$ in the FC limit). Therefore it is not clear at all how to define a work or an injected power and, consequently, a candidate for the entropy production. A theory for the fluctuations of $\Delta \theta$ in such a situation is, up to our knowledge, unknown and the discovery of the validity of the $\mathrm{FR}$ with a slope similar to a very different regime is largely unanticipated. We mention that in the Gaussian approximation, i.e. assuming a parabolic form for the large deviation rate of $\Delta \theta$ or equivalently

$$
f(\Delta \theta) \sim \exp \left[-\frac{(\Delta \theta-\langle\omega\rangle \Delta t)^{2}}{2 D \Delta t}\right]
$$

leads to the identification $s=2\langle\omega\rangle / D$. Again, no theoretical expectations exist, for the stochastic process modeled in Eq. (3), for the ratio between the average drift and the angular "diffusion" coefficient $D$. The empirical observation that such a ratio is somehow independent from the relative importance between collisions and dry friction (controlled by $\beta^{-1}$ ) is quite an interesting fact. Interestingly, in a previous paper [23] where similar measurements have been done on the different setup cited in the introduction [8], the validity of the FR for the asymmetry function $\phi(\Delta \theta)$ was observed at very different shaking strengths, with a slope $\sim 0.2$ independent from the dynamical regime (see Fig. 2a and Fig. 3b of that paper) and amazingly close to the slope measured in our experiment.

\section{CONCLUSIONS}

In summary we have repeated the studies recently appeared in [8] and 23], concerning the experimental measurement of the average Brownian motor effect and the analysis of the FR respectively, for a new setup which appears to be simple enough for a reasonable comparison against kinetic theory. Such a theory predicts two main regimes, rare collisions and frequent collisions, with two different formula for the average angular velocity of the rotator, formula (14) and (11) respectively. The same theory is able, only in the FC, to predict the validity of the FR $\phi(\Delta \theta)=s \Delta \theta$ with an analytical formula for the slope $s$, which is in good agreement with the experiments in that regime. The interesting observation, detailed above, is that such a slope does not depend strongly upon $\beta^{-1}$, giving a similar value even in a regime where collisions are rare excitations followed by fast dissipation due to dry friction. Future investigation of this puzzling observation is in order, in particular through numerical simulations or further variations of the experimental setup.

\section{ACKNOWLEDGMENTS}

The authors acknowledge the support of the Italian MIUR under the grants: FIRB-IDEAS n. RBID08Z9JE, FIRBs n. RBFR081IUK and n. RBFR08M3P4, and PRIN n. 2009PYYZM5.
[1] P. Reinmann, Phys. Rep. 361, 57 (2002)

[2] P. Hanggi and F. Marchesoni, Rev. Mod. Phys. 81, 387 (2003)

[3] R. P. Feynman, R. B. Leighton, and M. Sands, The Feynman Lectures on Physics (Addison-Wesley, Reading, MA, 1963)

[4] C. Van den Broeck, R. Kawai, and P. Meurs, Phys. Rev. Lett. 93, 090601 (2004)

[5] P. Meurs, C. Van den Broeck, and A. Garcia, Phys. Rev. E 70, 051109 (2004)
[6] C. Van den Broeck, P. Meurs, and R. Kawai, New J. Phys. 7, 10 (2005)

[7] Z. Farkas, P. Tegzes, A. Vukics, and T. Vicsek, Phys. Rev. E 60, 7022 (1999)

[8] P. Eshuis, K. van der Weele, D. Lohse, and D. van der Meer, Phys. Rev. Lett. 104, 248001 (2010)

[9] R. Balzan, F. Dalton, V. Loreto, A. Petri, and G. Pontuale, Phys. Rev. E 83, 031310 (2011)

[10] M. Heckel, P. Müller, T. Pöschel, and J. A. C. Gallas, Phys. Rev. E 86, 061310 (2012) 
[11] B. Cleuren and C. Van den Broeck, Europhys. Lett. 77, 50003 (2007)

[12] G. Costantini, A. Puglisi, and U. Marini Bettolo Marconi, Phys. Rev. E 75, 061124 (2007)

[13] B. Cleuren and R. Eichhorn, J. Stat. Mech., P10011(2008)

[14] G. Costantini, A. Puglisi, and U. Marini Bettolo Marconi, JSTAT in press (2009)

[15] J. Talbot, R. D. Wildman, and P. Viot, Phys. Rev. Lett. 107, 138001 (2011)

[16] J. Talbot, A. Burdeau, and P. Viot, J. Stat. Mech., P03009(2011)

[17] A. Gnoli, A. Petri, F. Dalton, G. Pontuale, G. Gradenigo, A. Sarracino, and A. Puglisi, Phys. Rev. Lett. 110, 120601 (2013)

[18] A. Sarracino, A. Gnoli, and A. Puglisi, "Ratchet effect driven by coulomb friction: the asymmetric rayleigh piston," Submitted (arXiv:1303.0700)

[19] P. S. Goohpattader and M. K. Chaudhury, J. Chem. Phys. 133, 024702 (2010)

[20] A. Baule and P. Sollich, EPL 97, 20001 (2012)
[21] U. Marini Bettolo Marconi, A. Puglisi, L. Rondoni, and A. Vulpiani, Phys. Rep. 461, 111 (2008)

[22] T. Hatano and S. Sasa, Phys. Rev. Lett. 86, 3463 (2001)

[23] S. Joubaud, D. Lohse, and D. van der Meer, Phys. Rev. Lett. 108, 210604 (2012)

[24] A. Naert, EPL 97, 20010 (2012)

[25] A. Mounier and A. Naert, EPL 100, 30002 (2012)

[26] A. Puglisi, A. Gnoli, G. Gradenigo, A. Sarracino, and D. Villamaina, J. Chem. Phys. 136, 014704 (2012)

[27] V. Kumaran, Phys. Rev. E 57, 5660 (1998)

[28] A. Buguin, F. Brochard, and P. G. de Gennes, Eur. Phys. J. E 19, 31 (2006)

[29] J. L. Lebowitz and H. Spohn, J. Stat. Phys. 95, 333 (1999)

[30] U. Seifert, Phys. Rev. Lett. 95, 040602 (2005)

[31] J. Talbot and P. Viot, Phys. Rev. E 85, 021310 (2012)

[32] A. Puglisi, P. Visco, E. Trizac, and F. van Wijland, Phys. Rev. E 73, 021301 (2006)

[33] A. Puglisi, L. Rondoni, and A. Vulpiani, J. Stat. Mech., P08010(2006) 